\title{
Safety of Monitored Anesthesia Care Using Propofol-Based Sedation for Pleuroscopy
}

\author{
Erik Vakil Mona Sarkiss David Ost Macarena R. Vial Roberto F. Casal \\ Georgie A. Eapen Horiana B. Grosu

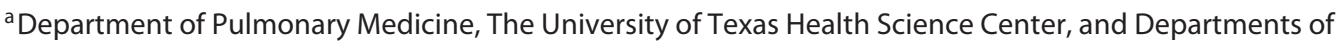 \\ ${ }^{b}$ Anesthesiology, ${ }^{c}$ Pulmonary Medicine and ${ }^{\mathrm{d} C y t o p a t h o l o g y, ~ T h e ~ U n i v e r s i t y ~ o f ~ T e x a s ~ M D ~ A n d e r s o n ~ C a n c e r ~ C e n t e r, ~}$ \\ Houston, TX, USA
}

\section{Keywords}

Pleuroscopy · Pleural biopsy · Pleural effusion · Anesthesia ·

Pleural disease - Propofol

\begin{abstract}
Background: The optimal approach to sedation for pleuroscopy remains undefined. Propofol is the favored sedativehypnotic for many proceduralists but has a narrow therapeutic window and the risk for oversedation is high. Propofolbased sedation administered by anesthesiologists and the routine use of end-tidal capnography and bispectral index (BIS) monitoring may attenuate risks of complications. $\mathbf{O b}$ jectives: The purpose of our study was to evaluate the safety and efficacy of monitored anesthesia care for pleuroscopy. Methods: We conducted a retrospective cohort study of patients who underwent pleuroscopy. The primary outcome of interest was the incidence of anesthesia complications in patients undergoing pleuroscopy. Hypoxia was defined as oxygen saturation of less than $90 \%$ for 2 min and hypotension was defined as the need for vasopressors. Results: Of 199 enrolled patients, there were no significant complications attributed directly to anesthesia. Minor complications included hypoxia in 9 patients (4.5\%), hypotension in 76 patients (38.2\%), and insertion of a nasopharyngeal tube air-
\end{abstract}

way in 2 patients (1.0\%). There was no significant difference in anesthesia-related complications between those with BIS monitoring and those without. Lower mean oxygen saturations ( $p=0.028)$ and hypoxia $(p=0.021)$ were found in patients receiving the combination of propofol plus narcotics plus sedatives compared to those receiving propofol only, propofol plus narcotics or propofol plus sedatives. Conclusion: Our study demonstrates that pleuroscopy using propofol with end-tidal capnography monitoring, with or without BIS monitoring, is safe and effective. The combination of propofol with narcotics and sedatives is associated with more hypoxia and lower mean oxygen saturation compared with propofol alone, propofol plus narcotics or propofol plus sedatives.

๑) 2017 S. Karger AG, Basel

\section{Introduction}

Pleuroscopy is the procedure of choice for diagnosing an exudative pleural effusion of unknown etiology [1-3]. Unlike video-assisted thoracoscopic surgery, pleuroscopy can be performed on an outpatient basis, does not require selective lung ventilation, and only requires moderate to deep sedation, rather than general anesthesia.

\section{KARGER}

(c) 2017 S. Karger AG, Basel

E-Mail karger@karger.com

www.karger.com/res
Horiana B. Grosu

Department of Pulmonary Medicine, Unit 1462

The University of Texas MD Anderson Cancer Center

1515 Holcombe Blvd., Houston, TX 77030 (USA)

E-Mail hbgrosu@mdanderson.org 
The optimal approach to moderate or deep sedation during pleuroscopy remains undefined. Monitored anesthesia care (MAC) allows improved monitoring, improved safety, and optimized sedation. However, the majority of experience with MAC has been in gastrointestinal endoscopy, where it has been found to be safe and effective [4].

Propofol has emerged as the drug of choice for MAC, as it has favorable sedative-hypnotic properties, a short half-life, and improved patient satisfaction scores compared with midazolam. However, the depth of sedation is less predictable with propofol and deep sedation may be achieved rapidly, increasing the risk for hypoventilation and apnea [5]. End-tidal capnography and bispectral index (BIS) may attenuate these risks and are a routine part of MAC. A study evaluating the role of end-tidal capnography during advanced gastrointestinal endoscopy showed that adding capnography to standard monitoring during procedural sedation significantly increases the detection of adverse respiratory events such as respiratory depression, apnea, oxygen desaturation, airway obstruction, and the need for oxygen supplementation [6].

A randomized trial by Grendelmeier et al. [7] compared midazolam with propofol during pleuroscopy administered by nonanesthesia personnel. Midazolam was associated with less hypotension and hypoxia than propofol. However, neither end-tidal capnography nor BIS monitoring was used during the procedure. A multicenter randomized trial initiated by Vorster et al. [8] comparing propofol/fentanyl with midazolam/fentanyl delivered by a nonspecialized anesthesiologist for pleuroscopy was terminated early after an interim analysis found the complication rate to be significantly greater in the propofol/fentanyl arm.

Conversely, Tschopp et al. [9] prospectively studied the use of propofol for pleuroscopy delivered by personnel who were trained by an anesthesia team prior to the start of the trial and who followed a strict administration protocol. They reported fewer adverse events than did Grendelmeier et al. [7]. The safety of propofol has also been well described for less invasive bronchoscopic procedures guided by nonanesthesia proceduralists [10-12].

At our institution, all pleuroscopies are performed with MAC with a goal of deep sedation. Propofol supported by end-tidal capnography is used in all cases. Additional sedatives, narcotics and BIS monitoring are used at the discretion of the anesthesiologist. The primary objective of our study was to evaluate the safety and efficacy of MAC for pleuroscopy using propofol and end-tidal capnography. We also evaluated whether the addition of
BIS monitoring had an impact on the incidence of anesthesia-related complications. We hypothesized that the incidence of anesthesia-related complications is lower in patients with BIS monitoring compared to those without.

\section{Methods}

\section{Population}

We performed a retrospective cohort study of consecutive patients aged 18 years or older who underwent pleuroscopy at The University of Texas MD Anderson Cancer Center from January 2005 to December 2015. Cases were identified using diagnostic codes in the MD Anderson clinical interactive reporting application. Institutional review board approval was obtained from the Institutional Review Board 4 Committee (protocol number PA160719). Patient demographic data, medical histories, laboratory findings, radiographic images, and anesthesia data on capnography, BIS monitoring, and procedure records were abstracted from their charts.

Complications were considered related to anesthesia if they were recorded at the time of the procedure or in the immediate post-procedure period. The following were the principle complications considered: hypoxia, hypotension, and need for ventilator support. Hypoxia was defined as an oxygen saturation of $<90 \%$, as measured by peripheral pulse oximetry $\left(\mathrm{SpO}_{2}\right)$ for $2 \mathrm{~min}$. Hypotension was defined as the need for vasopressors, which was at the discretion of the anesthesiologist. A need for ventilator support was defined as a clinical condition that required an advanced airway, laryngeal mask airway (LMA) or endotracheal tube, and initiation of mechanical ventilation.

Additional complications recorded per our institutional guidelines were an increase or decrease in heart rate by $50 \%$ from baseline, new cardiac arrhythmia requiring intervention, bag-valvemask ventilation, unplanned conversion to general anesthesia, abortion of the procedure after sedation, need for reversal agents, respiratory arrest, cardiac arrest, and death.

An escalation in the level of care was defined as admission to the hospital for outpatients or transfer to the intensive care unit for inpatients.

\section{Procedure Location and Designation}

All cases were performed in a dedicated pulmonary endoscopy suite with full anesthesia capabilities. The procedure was considered an outpatient procedure if it was performed on a prescheduled date and the patient was anticipated to be discharged the same day. Patients who underwent pleuroscopy with talc pleurodesis were considered inpatients since admission to the hospital after the procedure was anticipated. All other inpatient procedures were performed in patients who had already been admitted to the hospital for reasons not exclusively related to the need for pleuroscopy.

\section{Procedure Technique}

All pleuroscopy procedures were performed by interventional pulmonologists using MAC for deep sedation as previously described [13]. Patients were positioned in the lateral decubitus position with the affected side up. Ultrasound was used to identify the entry site. In some situations, a Boutin blunt tip trocar was used to 
create a pneumothorax. Blunt scissors or Kelly forceps were used for blunt dissection. Typically, an 8- or 10-mm trocar was inserted. Once the fluid was aspirated and the pleural cavity examined, biopsy specimens were obtained from the parietal pleura. Biopsies were obtained with rigid forceps in all patients. At the end of the procedure, a tunneled indwelling pleural catheter or a 20 -Fr chest tube was placed.

\section{Sedation}

The patients were initially positioned supine, where American Association of Anesthesiologists (ASA) monitors and a peripheral intravenous catheter were placed. Patients were then placed in the lateral decubitus position for pleuroscopy. All patients had an endtidal capnometer and end-tidal $\mathrm{CO}_{2}$ was continuously displayed and recorded. The choice of additional monitoring such as BIS and the anesthesia technique used were based on the anesthesiologist's preference and individual patient needs. When endobronchial ultrasound was planned immediately following pleuroscopy, pleuroscopy was performed under deep sedation as described above and the patient was then turned supine, anesthesia was deepened, a muscle relaxant was given, and an LMA inserted. At the end of the combined procedure, the muscle relaxant was reversed and the patient was awakened from anesthesia before the LMA was removed.

\section{Statistical Considerations}

Descriptive statistics were used to summarize the demographic and clinical characteristics of all patients in the study. For demographic and clinical characteristics, we used mean and SD to describe continuous variables distributed normally. We used medians for nonnormally distributed data. We used frequencies for categorical data. Categorical data were compared using the Fisher exact test. Analysis of covariance models were fitted for multiple categories of means and outcomes. $p$ values of 0.05 were considered to be significant. All tests were two-sided.

\section{Results}

We reviewed 199 consecutive cases of pleuroscopy. The median age of patients was 66.7 years (range, 18.292.7 years) and 117 (59\%) were male (Table 1). Most patients, 172 (86\%), had an existing diagnosis of malignan$\mathrm{cy}$ at the time of pleuroscopy. Eighty-nine (52\%) patients had solid tumors other than lung cancer, $53(31.0 \%)$ had lung cancer, and 30 (17\%) had hematologic malignancies. Patients generally had a good performance status, with 166 (83\%) having an Eastern Cooperative Oncology Group performance status of $0-1$. Most patients were considered to have severe systemic disease according to the ASA physical status classification system by virtue of having active malignancy, with 178 (89\%) having a score of 3. One hundred and sixty-eight procedures (84.4\%) were performed on an outpatient basis.

We divided the patients into two categories: those who underwent pleuroscopy with MAC with BIS monitoring
Table 1. Patient characteristics

\begin{tabular}{lc}
\hline Median age (IQR, 25-75), years & $66.7(18.2-92.7)$ \\
Sex & \\
Female & $82(41.2)$ \\
Male & $117(59.0)$ \\
Race & \\
White & $163(82.0)$ \\
Black & $9(5.0)$ \\
Hispanic & $18(9.0)$ \\
History of malignancy & \\
No & $27(14.0)$ \\
Yes & $172(86.4)$ \\
Cancer diagnosis & $30(17.4)$ \\
Hematologic malignancy & $53(31.0)$ \\
Lung cancer & $89(52.0)$ \\
Solid nonlung cancer & $31(16.0)$ \\
Procedure performed as an outpatient \\
No & $168(84.4)$ \\
Yes & $10(5.0)$ \\
Talc pleurodesis & $189(95.0)$ \\
TIPC placement & $8(4.0)$ \\
Eastern Cooperative Oncology Group performance status \\
0 & $57(29.0)$ \\
1 & $109(55.0)$ \\
2 & $28(14.0)$ \\
3 & $5(3.0)$ \\
American Society of Anesthesia score & \\
2 & $13(7.0)$ \\
3 & $178(89.4)$ \\
4 & $80(20)$ \\
BIS monitoring & \\
No & \\
Yes & \\
\hline
\end{tabular}

Figures are $n(\%)$ unless indicated otherwise. TIPC, tunneled indwelling pleural catheter.

and those without. There were $40(20 \%)$ patients who had BIS monitoring during the procedure (Table 2). There was no significant difference in anesthesia-related complications between those with BIS monitoring and those without with respect to hypoxia $(p=0.388)$, hypotension with a need for vasopressors $(p=0.364)$, and insertion of a nasopharyngeal tube airway $(p=0.362)$. There was also no significant difference in mean $\mathrm{CO}_{2}$ by end-tidal capnography $(p=0.125)$ and mean oxygen saturation $(p=$ 0.491).

We further divided the patients into four categories based on the sedative and analgesia medications delivered during MAC. These included propofol only, propofol plus narcotics, propofol plus sedatives, and propofol plus narcotics and sedatives. 
Table 2. Anesthesia complications in patients with and without BIS monitoring

\begin{tabular}{|c|c|c|c|c|}
\hline Parameter & $\begin{array}{l}\text { All patients } \\
(n=199)\end{array}$ & $\begin{array}{l}\text { BIS present } \\
(n=40)\end{array}$ & $\begin{array}{l}\text { BIS absent } \\
(n=159)\end{array}$ & $p$ value \\
\hline Hypoxia $\left(\mathrm{SpO}_{2}<90 \%\right)$ & 9 & 3 & 6 & 0.388 \\
\hline Mean $\mathrm{SpO}_{2}(\mathrm{SD})$ & $96.7(3.6)$ & $96.7(3.6)$ & $96.3(3.2)$ & 0.491 \\
\hline Mean $\mathrm{CO}_{2}$ by end-tidal capnography (SD) & $38.8(12.2)$ & $36.2(8.18)$ & $39.5(12.9)$ & 0.125 \\
\hline Need for ventilator support & 1 & 1 & 0 & 0.201 \\
\hline Admitted to hospital after outpatient procedure & 1 & 1 & 0 & 0.201 \\
\hline
\end{tabular}

Table 3. Anesthesia complications based on anesthesia drugs used

\begin{tabular}{|c|c|c|c|c|c|}
\hline Parameter & $\begin{array}{l}\text { Propofol } \\
(n=32)\end{array}$ & $\begin{array}{l}\text { Propofol/narcotics } \\
(n=111)\end{array}$ & $\begin{array}{l}\text { Propofol/sedatives } \\
(n=19)\end{array}$ & $\begin{array}{l}\text { Propofol/narcotics/ } \\
\text { sedatives } \\
(n=37)\end{array}$ & $\begin{array}{l}p \\
\text { value }\end{array}$ \\
\hline Mean $\mathrm{SpO}_{2}(\mathrm{SD})$ & $97.7(2.9)$ & $96.65(3.6)$ & $97.8(2.6)$ & $95.4(4.3)$ & 0.028 \\
\hline Mean $\mathrm{CO}_{2}$ by end-tidal capnography (SD) & $37.5(15)$ & $39.7(9.7)$ & $32.0(11.6)$ & $40.5(15.3)$ & 0.053 \\
\hline
\end{tabular}

The most commonly used drug combination was propofol plus narcotics. Fentanyl was the most commonly chosen narcotic in combination with propofol, and was given to 111 patients (55\%). Propofol plus narcotics plus sedatives was the second most common combination and was given to 37 patients (18\%). Propofol was used as the only sedative in 32 patients (16\%) and propofol plus other sedatives without narcotics in $19(10 \%)$.

The most commonly used narcotic in all patients was fentanyl in 142 patients (71\%), followed by hydromorphone in 6 patients (3\%), morphine in 1 patient (1\%), and remifentanil in 1 patient $(1 \%)$.

We found a statistically significant difference in patients receiving the combination of propofol plus narcotics plus sedatives in terms of hypoxia $(p=0.021)$ and also lower mean oxygen saturation $(p=0.028)$. The mean $\mathrm{CO}_{2}$ by end-tidal capnography $(p=0.053)$ and need for vasopressors $(p=0.679)$ were not statistically different among the groups (Table 3 ).

Seventeen patients underwent placement of an advanced airway. In 8 cases, the LMA was inserted prior to the procedure, at the discretion of the anesthesiologist, and spontaneous ventilation with pressure support was initiated. In 7 patients, the advanced airway was placed at the end of pleuroscopy in anticipation of endobronchial ultrasound. One patient required an endotracheal tube for pleuroscopy because of a recent esophagectomy and increased risk for aspiration.

One patient experienced hemodynamic instability and airway obstruction secondary to a tension pneumothorax after access to the pleural space. An LMA was placed and ultimately an endotracheal tube inserted for definitive airway management. Placement of advanced airway and need for mechanical ventilation was considered a procedure complication unrelated to MAC.

\section{Discussion}

Our study demonstrates that MAC with propofol and end-tidal capnography for pleuroscopy, with or without BIS monitoring, is both safe and effective. Patients who received a combination of propofol plus narcotics plus additional sedatives were found to have a lower mean oxygen saturation and more hypoxic events compared with patients who received a different combination of medications. We hypothesize that our lower rates of hypoxia and hypotension with propofol are in part due to MAC. MAC involves personnel who are trained specifically in the use of propofol and routinely use additional monitoring to 
optimize sedation delivery. Specifically, end-tidal capnography has been shown to be useful in titrating the depth of sedation in a variety of procedures that require sedation $[6,14-17]$.

The results of this study are similar to other studies evaluating end-tidal capnography during sedation in endoscopy $[6,18]$. A study by Qadeer et al. [6] randomly assigned patients undergoing elective endoscopic retrograde cholangiopancreatography and endoscopic ultrasonography to either a study arm, in which the endoscopy team was blinded to capnography, or an open arm, in which the endoscopy team was alerted to changes in endtidal $\mathrm{CO}_{2}$. Hypoxemia developed in $69 \%$ of patients in the blinded arm compared with $46 \%$ in the open arm $(p<$ 0.001 ) [6]. Although procedural sedation in this study was a combination of benzodiazepine and narcotic, it demonstrates the potential benefit of end-tidal $\mathrm{CO}_{2}$ monitoring.

To date, only one randomized trial has compared propofol with midazolam in pleuroscopy [7]. In this noninferiority trial, Grendelmeier et al. [7] randomly assigned 90 patients undergoing pleuroscopy to receive either midazolam delivered by intermittent bolus or propofol delivered by an initial bolus, followed by a continuous infusion. All sedation was administered by nonanesthesiologists with experience using propofol. The mean lowest oxygen saturation by $\mathrm{SpO}_{2}$ was significantly lower with propofol than with midazolam (93 \pm 6 vs. $96 \pm 3 \%$, $p=$ $0.007)$. The secondary endpoints of hypoxemia by arterial blood gas analysis ( 27 vs. $4 \%, p=0.007$ ) and hypotension by noninvasive cuff pressure measurement ( 82 vs. $40 \%, p<0.0001$ ) were also greater with propofol. In our study, only $5 \%$ of patients developed hypoxia and $38 \%$ required vasopressor support, which was a surrogate for clinically significant hypotension. There was no statistical difference between the groups receiving a different combination of sedatives and narcotics with respect to hypotension but a lower mean oxygen saturation and a higher number of hypoxic events were noted in those receiving propofol, a narcotic, and an additional sedative.

Our patient population was similar to the Grendelmeier cohort in terms of ASA scores, with ASA scores of 3 in 89 and $87 \%$ of patients, respectively. In our study, all patients underwent pleuroscopy for the diagnosis of a suspected malignant effusion or the treatment of a known malignant effusion. This is different to the Grendelmeier cohort, where only $48 \%$ were referred for effusions related to malignancy. It is unclear whether indication alone affects hypoxia and hypotension. However, the sedation protocol used in our study differs from the seda- tion protocol used by Grendelmeier and colleagues, where formal training was not provided and end-tidal capnography was not measured. All patients in our study were monitored with end-tidal capnography. Additional monitoring such as BIS was also used at the discretion of the anesthesiologist.

The potential benefits of training and additional monitoring were illustrated in a study by Tschopp et al. [9]. In this prospective cohort study of patients undergoing pleuroscopy with propofol, all personnel providing sedation were pretrained by experienced anesthesiologists on the proper use of propofol, a rigorous dosing schedule was implemented, and all patients were monitored using end-tidal capnography. Similar to our study, this paper reported lower rates of hypoxia and hypotension than those found by Grendelmeier and colleagues: 8 versus $27 \%$ and 64 versus $82 \%$, respectively.

The ASA recommends the involvement of an anesthesiologist in the care of patients undergoing deep sedation with propofol [19]. Propofol is also increasingly selected for endoscopic procedures due to its favorable pharmacokinetics, short-half life, and high satisfaction scores [20]. No studies have directly compared MAC with propofol-based sedation administered by nonanesthesiologists. Wadhwa et al. [4] performed a meta-analysis to compare the risks of propofol versus traditional anesthesia for gastrointestinal endoscopy. In a subgroup of 23 studies, MAC was noninferior for causing cardiopulmonary complications compared with propofol-based sedation administered by gastroenterologists (OR, 0.82; 95\% CI, 0.55-1.21).

Although the study of Wadhwa et al. [4] is intriguing, the broader applicability of their conclusions to pleuroscopy may be limited. A study by Chhajed et al. [21] demonstrated that pleuroscopy, performed under moderate sedation with midazolam, hydrocodone, and pethidine, was associated with significant hypoventilation (mean change in $\mathrm{PcCO}_{2}$ from a baseline of $13.2 \pm 5.3 \mathrm{~mm} \mathrm{Hg}$ ). A study by Trca et al. [22] showed that surgery of the pleural cavity is associated with significant hemodynamic alterations as a result of patient position and iatrogenic pneumothorax. Furthermore, patients undergoing pleuroscopy, by definition, have existing pulmonary disease, putting them at increased risk of respiratory events during sedation. Given these higher potential risks, additional monitoring and expertise by anesthesiologists may provide greater benefit in pleuroscopy compared with gastrointestinal endoscopy.

Interestingly, Vorster et al. [8] reported on an aborted multicenter randomized trial comparing propofol/fen- 
tanyl with midazolam/fentanyl for pleuroscopy. Sedation was delivered by a nonspecialist anesthesiologist. The trial was terminated after an interim analysis demonstrated higher complication rates in the propofol/fentanyl arm. This is in contrast to our study where the support of an anesthesiologist appears to mitigate complications. In the protocol by Vorster et al. [8], depth of sedation was measured by the Modified Observer's Assessment of Alertness/Sedation Scale and the use of additional monitoring devices such as end-tidal capnography or BIS monitoring was not reported. Although the Modified Observer's Assessment of Alertness/Sedation Scale is a validated sedation scale, it is more commonly used in the intensive care unit setting for patients receiving nonprocedural sedation. It is possible that the combination propofol boluses and an insensitive measure of sedation depth contributed to the higher than expected complication rate in the propofol/fentanyl arm.

It is noteworthy that 16 patients in our study had undergone advanced airway placement and mechanical ventilation during the procedure. The use of MAC may have provided increased flexibility in patients undergoing more than one procedure, may have mitigated complications in high-risk patients, and facilitated emergent intervention in patients with hemodynamic and respiratory compromise. Although these patients only represented $8 \%$ of our total cohort, MAC may have offered an additional benefit.

We recognize several limitations to our study including those inherent to retrospective cohort analyses. Our patients are not directly comparable to those in noncancer hospitals and our results may be confounded by an indication bias. Although the ASA scores of our patients were similar to those in other studies, the simplicity of the score undermines the potentially significant differences in patient characteristics that may affect sedation. For example, despite almost all of our patients having ASA scores of 3, the vast majority had an Eastern Cooperative Oncology Group performance status of $0-1$. This may not be the case in a patient with an empyema.

Our study also included patients who underwent pleuroscopy between 2005 and 2015. Over the same time period, there has been a significant evolution in the practice of pleuroscopy at our institution. This includes the experience of practitioners (both proceduralists and anesthesiologists), the location of the procedure (an operating room vs. a dedicated endoscopy suite), and the sedatives used (propofol alone or in combination with other sedatives). Therefore, important differences may exist within our own data depending on the year of treatment. That said, it is likely that safety and efficacy improved over the same time frame and that the inclusion of patients from many years ago would bias our results towards less favorable outcomes.

In conclusion, this is a relatively large study evaluating MAC for deep sedation during pleuroscopy using multiple drug combinations. Our data suggest that patients who undergo pleuroscopy with MAC have an acceptably low incidence of anesthesia-related complications. We also hypothesize that routine collaboration with anesthesiologists for propofol-based sedation during pleuroscopy may be beneficial. Additional caution should be taken when propofol is combined with a narcotic and an additional sedative to achieve deep sedation as the patient may be at greater risk for hypoxia.

\section{Acknowledgements}

No funding was received.

\section{Financial Disclosure and Conflicts of Interest}

The authors have no conflicts of interest.

\section{References}

1 Blanc FX, Atassi K, Bignon J, Housset B: Diagnostic value of medical thoracoscopy in pleural disease: a 6-year retrospective study. Chest 2002;121:1677-1683.

2 DePew ZS, Wigle D, Mullon JJ, Nichols FC, Deschamps C, Maldonado F: Feasibility and safety of outpatient medical thoracoscopy at a large tertiary medical center: a collaborative medical-surgical initiative. Chest 2014;146: 398-405.
3 Hansen M, Faurschou P, Clementsen P: Medical thoracoscopy, results and complications in 146 patients: a retrospective study. Respir Med 1998;92:228-232.

4 Wadhwa V, Issa D, Garg S, Lopez R, Sanaka MR, Vargo JJ: Similar risk of cardiopulmonary adverse events between propofol and traditional anesthesia for gastrointestinal endoscopy: a systematic review and meta-analysis. Clin Gastroenterol Hepatol 2017;15: 194-206.

\footnotetext{
5 Riphaus A, Gstettenbauer T, Frenz MB, Wehrmann T: Quality of psychomotor recovery after propofol sedation for routine endoscopy: a randomized and controlled study. Endoscopy 2006;38:677-683.

6 Qadeer MA, Vargo JJ, Dumot JA, et al: Capnographic monitoring of respiratory activity improves safety of sedation for endoscopic cholangiopancreatography and ultrasonography. Gastroenterology 2009;136:1568-1576; quiz 1819-1820.
} 
7 Grendelmeier P, Tamm M, Jahn K, Pflimlin E, Stolz D: Propofol versus midazolam in medical thoracoscopy: a randomized, noninferiority trial. Respiration 2014;88:126-136.

8 Vorster MJ, Bruwer JW, Frank W, Bernasconi M, Koegelenberg C: The use of propofol for sedation in medical thoracoscopy. Respiration 2015;89:435.

9 Tschopp JM, Purek L, Frey JG, et al: Titrated sedation with propofol for medical thoracoscopy: a feasibility and safety study. Respiration 2011;82:451-457.

10 Clark G, Licker M, Younossian AB, et al: Titrated sedation with propofol or midazolam for flexible bronchoscopy: a randomised trial. Eur Respir J 2009;34:1277-1283.

11 Bosslet GT, Devito ML, Lahm T, Sheski FD, Mathur PN: Nurse-administered propofol sedation: feasibility and safety in bronchoscopy. Respiration 2010;79:315-321.

12 Stolz D, Kurer G, Meyer A, et al: Propofol versus combined sedation in flexible bronchoscopy: a randomised non-inferiority trial. Eur Respir J 2009;34:1024-1030.
13 Ghisi D, Fanelli A, Tosi M, Nuzzi M, Fanelli G: Monitored anesthesia care. Minerva Anestesiol 2005; 71:533-538.

14 Ishiwata T, Tsushima K, Fujie M, et al: Endtidal capnographic monitoring to detect apnea episodes during flexible bronchoscopy under sedation. BMC Pulm Med 2017;17:7.

15 Deitch K, Miner J, Chudnofsky CR, Dominici $\mathrm{P}$, Latta D: Does end tidal $\mathrm{CO}_{2}$ monitoring during emergency department procedural sedation and analgesia with propofol decrease the incidence of hypoxic events? A randomized, controlled trial. Ann Emerg Med 2010; 55:258-264.

16 Lo Y-L, Lin T-Y, Fang Y-F, et al: Feasibility of bispectral index-guided propofol infusion for flexible bronchoscopy sedation: a randomized controlled trial. PLoS One 2011;6:e27769.

17 Fadaizadeh L, Hoseyni MS, Shajareh E, Heydari G, Ardehali SH: Use of bispectral index score for interventional bronchoscopy procedures. Tanaffos 2015;14:246-251.
18 Beitz A, Riphaus A, Meining A, et al: Capnographic monitoring reduces the incidence of arterial oxygen desaturation and hypoxemia during propofol sedation for colonoscopy: a randomized, controlled study (ColoCap Study). Am J Gastroenterol 2012;107:12051212.

19 American Society of Anesthesiologists: Statement of safe use of propofol. 2014. http:// www.asahq.org/ /media/sites/asahq/files/ public/resources/standards-guidelines/statement-on-safe-use-of-propofol.pdf.

20 José RJ, Shaefi S, Navani N: Sedation for flexible bronchoscopy: current and emerging evidence. Eur Respir Rev 2013;22:106.

21 Chhajed PN, Kaegi B, Rajasekaran R, Tamm M: Detection of hypoventilation during thoracoscopy: combined cutaneous carbon dioxide tension and oximetry monitoring with a new digital sensor. Chest 2005; 127:585-588.

22 Trca S, Krska Z, Kittnar O, et al: Hemodynamic response to thoracoscopy and thoracotomy. Physiol Res 2010;59:363-371. 